\title{
Application of Green Fluorescent Protein in Immunoassays
}

\author{
Seiichi Sakamoto1*, Yukihiro Shoyama ${ }^{2}$, Hiroyuki Tanaka1, Satoshi Morimoto1 \\ ${ }^{1}$ Department of Pharmacognosy, Graduate School of Pharmaceutical Sciences, Kyushu University, Fukuoka, \\ Japan \\ ${ }^{2}$ Department of Pharmacognosy, Faculty of Pharmaceutical Sciences, Nagasaki International University, \\ Nagasaki, Japan \\ Email: s.sakamoto@phar.kyushu-u.ac.jp, shoyama@niu.ac.jp, htanaka@phar.kyushu-u.ac.jp, \\ morimoto@phar.kyushu-u.ac.jp
}

Received 18 February 2014; revised 26 April 2014; accepted 15 May 2014

Copyright (C) 2014 by authors and Scientific Research Publishing Inc.

This work is licensed under the Creative Commons Attribution International License (CC BY). http://creativecommons.org/licenses/by/4.0/

(c) (i) Open Access

\begin{abstract}
Green fluorescent protein (GFP) is a protein that emits green fluorescence when exposed to a radiation of ultraviolet wavelength range, even without the addition of substrate and cofactor. Because of such characteristics, the usage of GFP is widespread in both in vivo and in vitro applications. In addition, recent advances in biotechnology have enabled GFP to be expressed in various hosts, including bacteria, yeast, plants, animals, and even living-cells, for multiple purposes. Currently, GFP is a subject of great interest in the analytical sciences, especially in immunoassays for qualitative and quantitative analyses, when it is fused with an antibody because of the high sensitivity of GFP and antigen-binding specificity of antibodies. Recently the fluobody, which is a fusion protein of GFP with single-chain variable fragment antibody (scFv), has become a useful tool in various fields. We review here the applications of GFP as fluobodies in immunoassays.
\end{abstract}

\section{Keywords}

GFP, Immunoassay, Fluobody

\section{Introduction}

Green fluorescent protein (GFP) was first isolated from the jellyfish Aequorea victoria in the early 1960s [1]. The fluorescence spectra of GFP exhibit two absorption peaks at $395 \mathrm{~nm}$ and $475 \mathrm{~nm}$ (excitation), which individually emitted maximum at $508 \mathrm{~nm}$ and $503 \mathrm{~nm}$ (emission). The absorption peaks at $395 \mathrm{~nm}$ and $475 \mathrm{~nm}$ re-

\footnotetext{
*Corresponding author.
}

How to cite this paper: Sakamoto, S., Shoyama, Y., Tanaka, H. and Morimoto, S. (2014) Application of Green Fluorescent Protein in Immunoassays. Advances in Bioscience and Biotechnology, 5, 557-563. 
sult from two different chromophore species: a neutral protonated form and an anionic deprotonated form, respectively [2] [3]. The primary structure of GFP was elucidated three decades after its first discovery, and the GFP gene was found to encode 238 amino acid residues with a calculated molecular weight of 26,888 Da [4]. Thereafter, the use of GFP increased widely and dramatically. The first expression of GFP was successfully performed in nematode Caenorhabditis elegans [5], and the results suggested that GFP can be used as a marker for gene expression. In 1996, two groups successfully crystallized GFP and found that the protein fold consists of an 11-stranded $\beta$-barrel with a coaxial helix, with the chromophore forming from the central helix [6] [7]. Currently, GFP is an indispensable tool as a reporter gene for the study of gene expression and subcellular localization of proteins in plant, bacterial, and mammalian systems [8]-[10]. This widespread use of GFP as a biological tool is attributed to three characteristics. First, GFP is highly stable. The fluorescence of GFP is species-independent and remains stable under a wide variety of conditions [11]. Second, GFP maturation requires only oxygen [12]. Third, GFP is noninvasive, and therefore the fluorescence can be monitored in living cells, or in the entire organism in case of transparent organisms [13].

Thus far, various applications of GFP in both in vivo direct monitoring of the target protein and in vitro analyses have been reported. Fluorescence resonance energy transfer (FRET) is one of the most widely used applications of GFP. FRET is the nonradiative exchange of energy from an excited donor fluorophore to an acceptor fluorophore within 10 - $100 \AA$. Because the efficiency of FRET depends on the distance between fluorophores, protein-protein interactions can be investigated using this method in vivo and in vitro. GFP can be used as an intercellular molecular sensor in this case. The applications of GFP in FRET have recently been reviewed [14]-[18]. The use of GFP has been expanded to photobleaching, which can be used to explore the lateral mobility of fluorophores on surface of cells. The fusion of GFP with a target protein makes it possible to investigate the protein dynamics within a cell [19]-[21]. Two different types of photobleaching processes can be used to explore molecular motion or diffusion: fluorescence recovery after photobleaching (FRAP) and fluorescence loss in photobleaching (FLIP). In the FRAP method, a region of interest is bleached once by high-intensity illumination, and then recovery of the resultant fluorescence loss is monitored, which reveals the relative mobility of the GFP chimera protein [20]. FLIP can be used to study the transport of GFP chimera proteins between different organelles. After a region has been repeatedly bleached, the loss of fluorescence in an area away from the bleached region is monitored. The FLIP method can be used to confirm FRAP results because the photobleaching process in FRAP can photo damage the protein of interest and alter its dynamic characteristics, whereas FLIP assesses the mobility of fluorescent proteins adjacent to the bleached region [22].

A fluobody, which is a chimera protein of GFP combined with antibody, has become a useful tool in both in vivo and in vitro applications. In in vivo applications, a fluobody can be used to "tag" the target protein by utilizing binding specificity of an antibody when the fluobody is expressed in a living host. Li et al. recently found that the camelid-derived homodimeric heavy-chain antibody (VHH), which is composed of a single-variable domain, can across the blood-brain barrier (BBB) without any treatment [23]. The VHH antibodies against the astrocyte-specific marker-human glial fibrillary acidic protein (GFAP), were genetically fused with GFP to produce a fluobody, and this fluobody was expressed to label the GFAP in murine brain. As a result, the fluobody could pass the BBB and label GFAP. This study revealed the ability of the fluobody to be used as a specific transporter. Another application has been demonstrated in plants [24], where the investigators constructed a fluobody directly against phytohormone, which is a precursor of gibberellin (GA24) that regulates growth and influences various developmental processes [25]. This fluobody was expressed in Arabidopsis thaliana. The results revealed the localization of scFv, which is bound to GA24. In this case, the fluobody was applied as an in vivo tool to elucidate the mechanism of the appearance of a GA-deficient phynotype. When the scFv antibody targets the cancer-cell receptor, which is associated with cell proliferation, it can be used in molecular targeted therapy [26]-[28]. Huang et al. constructed a fluobody against HepG2 hepatocellular carcinoma and injected the fluobody into mice to elucidate the localization in HepG2 [29]. The fluorescence derived from the fluobody was detected 24 hours after injection, which demonstrates the potential use of fluobodies in in vivo molecular targeted therapy.

With respect to in vitro applications, fluobodies have been widely used as probes in immunoassays for qualitative/quantitative analysis. In an analytical application, a highly sensitive probe is generally required; thus, the conjugation between fluorescent labels and antibodies has conventionally been performed by chemical conjugation of organic fluorophores [30]; fluorescein isothiocyanate (FITC) is the most commonly employed fluorophore in various fields. Because FITC can bind to the free amino group of the proteins and peptides to form sta- 
ble thiourea bond, FITC-labeled antibodies have been used primarily for flow cytometry and immunohistochemical staining for more than 50 years [31] [32]. This procedure, however, requires a large amount of purified protein. Moreover, the fluorophores may bind to the paratope of an antibody, which results in the antibody's partial or complete loss of reactivity. A fluobody can overcome these disadvantages because once the genes are constructed in the expression vector, the resultant protein is always expressed in a one-to-one ratio between the fluorochrome and scFv. Currently, the usage of fluobodies in vitro has expanded from the immunolabeling of cancer cells, fluorescence-activated cell sorter (FACS), and diagnosis, to fluorescent-linked immunosorbent assay (FLISA) for the qualitative/quantitative analyses [33]-[37].

The in vitro applications of GFP as a fluobody are reviewed in this article.

\section{Applications of Fluobody in Immunoassays}

\subsection{Immunolabeling}

The fluobody possesses two characteristics: strong emission intensity and an antibody that binds to a target antigen. Immunolabeling using fluobodies is convenient method for investigating the subcellular protein and oncoprotein in living cells. When the cells are treated with the fluobody and washed, they are ready to be observed under a fluorescence microscope. Schwalbach et al. successfully expressed a fluobody against the E6 protein of human papillomavirus type 16 (E6 protein) in Escherichia coli (E. coli) and revealed that the subcellular localization and movement of E6 protein transfected in COS-7 cell lines [38]. Around the same period, Casey et al. expressed a fluobody specific to hepatitis B surface antigen (HepBsAg) in the periplasmic fraction of E. coli and revealed that the fluobody retains the functional form to recognize HepBsAg even though it is expressed as a periplasmic protein [33]. In addition, a fluobody against a series of cluster of differentiation (CD) antigen [35], p24 (human immunodeficiency virus 1) CB4-1 [39], single-chain T-cell receptor (scTCR) [40], and B-cell-activating factor of the TNF family (BAFF) [36] were successfully applied for one-step immunolabeling. More recently, a new, multi-colored newly fluobody was suggested by Markiv et al. [41]. They constructed a fluorescent antibody, where humanized scFv against p185 ${ }^{\text {HER-2-ECD }}$ (Trastuzumab, Herceptin, 4D5), which is widely used in breast cancer immunotherapy [42], was fused with four kinds of monomeric red, blue, cerulean and citrine fluorescent proteins (RFP, BFP, CER, and CIT, respectively) as bridging molecules. A cell staining study using SK-BR-3 breast carcinoma cells revealed that 4D5-RFP and 4D5-CIT were found to specifically recognize $185^{\text {HER-2-ECD }}$ and effectively accumulate on the surface of the cells. Notably, a fluorescent protein other than GFP was used as a fluorescent probe. The results of this study open up the possibility of fluobodies that can be used in immunolabeling because of the various measurement wavelengths of fluorochrome.

\subsection{Fluorescence-Activated Cell Sorter}

Peipp et al. prepared scFv fused with two fluorescent proteins (GFP and DsRed); the fused product exhibited spectral properties that are ideal for dual-color experiments with GFP [35] [43] in which it is applied as a fluorescence-activated cell sorter (FACS). One common problem frequently encountered with a FACS is the background associated with the antigen-independent interaction between the Fc-protein and Fc receptors on various cells. These interactions result in a diminished intensity of the specific signal and subsequently cause a decrease in the signal-to-noise ratio, which complicates the collection of experimental date [44] [45]. Therefore, Peipp et al. used a fluorescent antibody because scFv antibody is composed of minimal variable regions for antigenbinding activity without Fc regions. They successfully reduced the background and enhanced the signal-tonoise ratio by a factor of 5 - 10 compared with the value obtained using conventional antibodies. Their study demonstrates the possible use of fluobodies in FACS, especially when treating with cell populations expressing Fc receptors.

\subsection{Fluorescence-Linked Immunosorbent Assay}

FLISA can be used for both large molecules and small molecules [46] [47]. Indirect FLISA (iFLISA) is suitable for large molecules such as proteins and peptides, whereas indirect competitive FLISA (icFLISA) is appropriate for small molecules such as natural products, agrochemicals, pesticides, and herbicides. The advantages of FLISA over the conventional enzyme-linked immunosorbent assay (ELISA) are its rapidity and sensitivity. Conventional ELISA using an antibody (i.e., monoclonal antibody, polyclonal antibody, or scFv antibody) re- 
quires the following five steps, and the assay requires approximately 4.5 hours: 1) fixation of a coated antigen, 2) a blocking step to prevent the plate from adsorbing non-specific proteins, 3) the primary antibody reaction, 4) the secondary antibody reaction, and 5) the enzyme-substrate reaction. However, in the case of FLISA performed using a fluobody, the assay is completed within 3 hours because the time-consuming and costly secondary antibody reaction and the subsequent enzyme-substrate reaction can be avoided. In addition, sensitive FLISA can be performed by taking advantage of the strong fluorescence intensity of GFP [48]. Up to date, icFLISA can be developed to detect/determine small molecules, which include picloram [49], s-triazine [50], 5-methyl 2'-deoxycytidine [51] and bioactive natural product, plumbagin (PL) and ginsenoside Re (GRe) as previously described in our group [52]-[54]. The icFLISA for PL and GRe shows a lower limit of determination than conventional icELISA. In our study, we assessed the formation of a fluobody has been assessed by constructing two chimera proteins of scFv fused at the C-terminus of GFP (C-fluobody) and the N-terminus of GFP ( $\mathrm{N}$-fluobody). In both cases, the fluorescence intensity of the C-fluobody was superior to that of the $\mathrm{N}$-fluobody. These results are supported by the findings of other groups [33] [51]. The difference in fluorescence intensity between the two can be accounted for the flexibility of the linker peptide fusing GFP and the scFv antibody. The 10 amino acids (30 bp) of the C-terminus of the GFP are well known to be flexible sequence [6] and can function as an additional linker in the C-fluobody fusing scFv at the C-terminus of GFP. Because the length of the linker peptide of fluobody was designed to contain 10 amino acids of $\left(\mathrm{Gly}_{4} \mathrm{Ser}\right)_{2}$ encoded by $30 \mathrm{bp}$, the linker sequence of the C-fluobody is equivalent to twice as long as that of the N-fluobody. Our results showed that the flexibility of the linker strongly affects the function of GFP in the fluobody.

Recently, a new type of FLISA has been developed by Ferrara et al. [55]. In their fluobody, scFv was expressed with a tag composed of a small domain of GFP (strand 11, residues 215 - 230; GFP11) with retaining its antigen-binding activity. The complementary GFP fragment $(1-10$, residues 1 - 214) is expressed separately. Neither fragment alone is fluorescent. Only when they are mixed, the small and large GFP fragments spontaneously associate, resulting in reconstitution of the fluorophore and fluorescence. This approach allows both the antigen and the scFv concentrations to be determined.

\section{Conclusion}

In this review, the applications of GFP as a fluobody have been described. Although the fluobody was defined as a chimera protein of GFP with ScFv in this article, variations of fluobodies can be designed for different purposes. The type of fluorescent protein used can be expanded from GFP to UV, blue, cyan, yellow, orange, and red fluorescent proteins. The varieties of known fluorescent proteins have been well reviewed [56]. In addition, the antibody can be changed from scFv to fragment antigen-binding (Fab) ( $\sim 55 \mathrm{kDa}), \mathrm{Fab}_{2}$ (bispecific; $\sim 110$ $\mathrm{kDa}$ ), $\mathrm{Fab}_{3}$ (trispecific; $\sim 165 \mathrm{kDa}$ ), diabody (bispecific; $\sim 50 \mathrm{kDa}$ ), triabody (trivalent; $\sim 75 \mathrm{kDa}$ ), tetrabody (tetravalent; 100 kDa), bis-scFv (bispecific; $\sim 55 \mathrm{kDa}$ ), and minibody (bivalent; $75 \mathrm{kDa}$ ) antibodies [57]. These combinations, in conjunction with different linker designs, open up the use of fluobodies more in various fields.

\section{Acknowledgements}

This work was funded by Grant-in-Aid for Young Scientists (B) of the Japan Society for the Promotion of Science (JSPS) KAKENHI Grant Number 25750167. This work was also funded by a Grant in Aid from the Japan Society for the Promotion of Science Asian CORE Program of the Ministry of Education, Culture, Sports, Science, and Technology of Japan.

\section{References}

[1] Shimomura, O., Johnson, F.H. and Saiga, Y. (1962) Extraction, Purification and Properties of Aequorin, a Bioluminescent Protein from the Luminous Hydromedusan, Aequorea. Journal of Cellular and Comparative Physiology, 59, 223-239. http://dx.doi.org/10.1002/jcp.1030590302

[2] Zimmer, M. (2002) Green Fluorescent Protein (GFP): Applications, Structure, and Related Photophysical Behavior. Chemical Reviews, 102, 759-781. http://dx.doi.org/10.1021/cr010142r

[3] Niwa, H., Inouye, S., Hirano, T., Matsuno, T., Kojima, S., Kubota, M., Ohashi, M. and Tsuji, F.I. (1996) Chemical Nature of the Light Emitter of the Aequorea Green Fluorescent Protein. Proceedings of the National Academy of Sciences of the United States of America, 93, 13617-13622. http://dx.doi.org/10.1073/pnas.93.24.13617

[4] Prasher, D.C., Eckenrode, V.K., Ward, W.W., Prendergast, F.G. and Cormier, M.J. (1992) Primary Structure of the 
Aequorea victoria Green-Fluorescent Protein. Gene, 111, 229-233. http://dx.doi.org/10.1016/0378-1119(92)90691-H

[5] Chalfie, M., Tu, Y., Euskirchen, G., Ward, W.W. and Prasher D.C. (1994) Green Fluorescent Protein as a Marker for Gene Expression. Science, 263, 802-805. http://dx.doi.org/10.1126/science.8303295

[6] Ormo, M., Cubitt, A.B., Kallio, K., Gross, L.A., Tsien, R.Y. and Remington, S.J. (1996) Crystal Structure of the Aequorea victoria Green Fluorescent Protein. Science, 273, 1392-1395.

[7] Yang, F., Moss, L.G. and Phillips, G.N.J. (1996) The Molecular Structure of Green Fluorescent Protein. Nature Biotechnology, 14, 1246-1251. http://dx.doi.org/10.1038/nbt1096-1246

[8] Sheen, J., Hwang, S.B., Niwa, Y., Kobayashi, H. and Galbraith, D.W. (1995) Green-Fluorescent Protein as a New Vital Marker in Plant Cells. Plant Journal, 8, 777-784. http://dx.doi.org/10.1046/j.1365-313X.1995.08050777.x

[9] Cormack, B.P., Valdivia, R.H. and Falkow, S. (1996) FACS-Optimized Mutants of the Green Fluorescent Protein (GFP). Gene, 173, 33-38. http://dx.doi.org/10.1016/0378-1119(95)00685-0

[10] Kain, S.R., Adams, M., Kondepudi, A., Yang, T.T., Ward, W.W. and Kitts, P. (1995) Green Fluorescent Protein as a Reporter of Gene Expression and Protein Localization. Biotechniques, 19, 650-655.

[11] Patterson, G.H., Knoble, S.M., Sharif, W.D., Kain, S.R. and Piston, D.W. (1997) Use of the Green Fluorescent Protein and Its Mutants in Fluorescence Microscopy. Biophysical Journal, 73, 2782-2790. http://dx.doi.org/10.1016/S0006-3495(97)78307-3

[12] Heim, R., Prasher, D.C. and Tsien, R.Y. (1994) Wavelength Mutation and Posttranslational Autoxidation of Green Fluorescent Protein. Proceedings of the National Academy of Sciences of the United States of America, 91, 1250112504. http://dx.doi.org/10.1073/pnas.91.26.12501

[13] Liu, H.S., Jan, M.S., Chou, C.K., Chen, P.H. and Ke, N.J. (1999) Is Green Fluorescent Protein Toxic to the Living Cells? Biochemical and Biophysical Research Communications, 260, 712-717. http://dx.doi.org/10.1006/bbrc.1999.0954

[14] Ueda, Y., Kwok, S. and Hayashi, Y. (2013) Application of FRET Probes in the Analysis of Neuronal Plasticity. Frontiers in Neural Circuits, 7, 163.

[15] Nouar, R., Devred, F., Breuzard, G. and Peyrot, V. (2013) FRET and FRAP Imaging: Approaches to Characterise Tau and Stathmin Interactions with Microtubules in Cells. Biology of the Cell, 105, 149-161. http://dx.doi.org/10.1111/boc.201200060

[16] Zeug, A., Woehler, A., Neher, E. and Ponimaskin, E.G. (2012) Quantitative Intensity-Based FRET Approaches-A Comparative Snapshot. Biophysical Journal, 103, 1821-1827. http://dx.doi.org/10.1016/j.bpj.2012.09.031

[17] Preus, S. and Wilhelmsson, L.M. (2012) Advances in Quantitative FRET-Based Methods for Studying Nucleic Acids. ChemBioChem, 13, 1990-2001. http://dx.doi.org/10.1002/cbic.201200400

[18] Galbán, J., Sanz-Vicente, I., Ortega, E., del Barrio, M. and de Marcos, S. (2012) Reagentless Fluorescent Biosensors Based on Proteins for Continuous Monitoring Systems. Analytical and Bioanalytical Chemistry, 402, 3039-3054. http://dx.doi.org/10.1007/s00216-012-5715-2

[19] White, J. and Stelzer, E. (1999) Photobleaching GFP Reveals Protein Dynamics Inside Live Cells. Trends in Cell Biology, 9, 61-65. http://dx.doi.org/10.1016/S0962-8924(98)01433-0

[20] Zimmer, M. (2002) Green Fluorescent Protein (GFP): Applications, Structure, and Related Photophysical Behavior. Chemical Reviews, 102, 759-781. http://dx.doi.org/10.1021/cr010142r

[21] Lippincott-Schwartz, J., Snapp, E. and Kenworthy, A. (2001) Studying Protein Dynamics in Living Cells. Nature Reviews Molecular Cell Biology, 2, 444-456. http://dx.doi.org/10.1038/35073068

[22] Goulbourne, C.N., Malhas, A.N., Doolittle, H., Ismail, A.T. and Vaux, D.J. (2010) Study of Molecular Dynamics Using Fluorescently Tagged Molecules in Live Cells. In: Méndez-Vilas, A. and Díaz, J., Eds., Microscopy: Science, Technology, Applications and Education, 670-678.

[23] Li, T., Bourgeois, J.P., Celli, S., Glacial, F., Le Sourd, A.M., Mecheri, S., Weksler, B., Romero, I., Couraud, P.O., Rougeon, F. and Lafaye, P. (2012) Cell-Penetrating Anti-GFAP VHH and Corresponding Fluorescent Fusion Protein VHH-GFP Spontaneously Cross the Blood-Brain Barrier and Specifically Recognize Astrocytes: Application to Brain Imaging. The FASEB Journal, 26, 3969-3979. http://dx.doi.org/10.1096/fj.11-201384

[24] Urakami, E., Yamaguchi, I., Asami, T., Conrad, U. and Suzuki, Y. (2008) Immunomodulation of Gibberellin Biosynthesis Using an Anti-Precursor Gibberellin Antibody Confers Gibberellin-Deficient Phenotypes. Planta, 228, 863-873. http://dx.doi.org/10.1007/s00425-008-0788-z

[25] Yamaguchi, S. (2008) Gibberellin Metabolism and Its Regulation. Annual Review of Plant Biology, 59, 225-251. http://dx.doi.org/10.1146/annurev.arplant.59.032607.092804

[26] Ahmad, Z.A., Yeap, S.K., Ali, A.M., Ho, W.Y., Alitheen, N.B. and Hamid, M. (2012) scFv Antibody: Principles and Clinical Application. Clinical \& Developmental Immunology, 2012, 980250. 
[27] Beckman, R.A., Weiner, L.M. and Davis, H.M. (2007) Antibody Constructs in Cancer Therapy: Protein Engineering Strategies to Improve Exposure in Solid Tumors. Cancer, 109, 170-179. http://dx.doi.org/10.1002/cncr.22402

[28] Leath 3rd, C.A., Douglas, J.T., Curiel, D.T. and Alvarez, R.D. (2004) Single-Chain Antibodies: A Therapeutic Modality for Cancer Gene Therapy (Review). International Journal of Oncology, 24, 765-771.

[29] Huang, J., Li, Y., Guo, F., Tong, Y., Wang, J., Hu, J. and Li, G. (2011) Expression of scFv SA3 against Hepatoma Fused with Enhanced Green Fluorescent Protein and Its Targeted Ability in Vivo. Zhong Nan Da Xue Xue Bao Yi Xue Ban/Journal of Central South University, 36, 979-986.

[30] Hermanson, G.T. (1996) Bioconjugate Techniques. Academic Press, San Diego, 297-364.

[31] Goldstein, G., Slizys, I.S. and Chase, M.W. (1961) Studies on Fluorescent Antibody Staining. I. Non-Specific Fluorescence with Fluorescein-Coupled Sheep Anti-Rabbit Globulins. The Journal of Experimental Medicine, 114, 89-110. http://dx.doi.org/10.1084/jem.114.1.89

[32] Sommerville, R.G. (1967) The Production of Fluorescent Antibody Reagents for Virus Diagnosis in the Albino Mouse. Archiv für die gesamte Virusforschung, 20, 452-458. http://dx.doi.org/10.1007/BF01275226

[33] Casey, J.L., Coley, A.M., Tilley, L.M. and Foley, M. (2000) Green Fluorescent Antibodies: Novel in Vitro Tools. Protein Engineering, 13, 445-452. http://dx.doi.org/10.1093/protein/13.6.445

[34] Souriu, C. and Hudson, P.J. (2001) Recombinant Antibodies for Cancer Diagnosis and Therapy. Expert Opinion on Biological Therapy, 1, 845-855. http://dx.doi.org/10.1517/14712598.1.5.845

[35] Peipp, M., Saul, D., Barbin, K., Bruenke, J., Zunino, S.J., Niederweis, M. and Fey, G.H. (2004) Efficient Eukaryotic Expression of Fluorescent scFv Fusion Proteins Directed against CD Antigens for FACS Applications. Journal of Immunological Methods, 285, 265-280. http://dx.doi.org/10.1016/j.jim.2003.12.001

[36] Cao, M., Cao, P., Yan, H.J., Ren, F., Lu, W.G., Hu, Y.L. and Zhang, S.Q. (2008) Construction and Characterization of an Enhanced GFP-Tagged Anti-BAFF scFv Antibody. Applied Microbiology and Biotechnology, 79, 423-431. http://dx.doi.org/10.1007/s00253-008-1447-9

[37] Olichon, A. and Surrey, T. (2007) Selection of Genetically Encoded Fluorescent Single Domain Antibodies Engineered for Efficient Expression in Escherichia coli. The Journal of Biological Chemistry, 282, 36314-36320. http://dx.doi.org/10.1074/jbc.M704908200

[38] Schwalbach, G., Sibler, A.P., Choulier, L., Deryckère, F. and Weiss, E. (2000) Production of Fluorescent Single-Chain Antibody Fragments in Escherichia coli. Protein Expression and Purifications, 18, 121-132. http://dx.doi.org/10.1006/prep.1999.1185

[39] Naumann, J.M., Küttner, G. and Bureik, M. (2011) Expression and Secretion of a CB4-1 scFv-GFP Fusion Protein by Fission Yeast. Applied Biochemistry and Biotechnology, 163, 80-89. http://dx.doi.org/10.1007/s12010-010-9018-9

[40] Huang, D. and Shusta, E.V. (2006) A Yeast Platform for the Production of Single-Chain Antibody-Green Fluorescent Protein Fusions. Applied and Environmental Microbiology, 72, 7748-7759. http://dx.doi.org/10.1128/AEM.01403-06

[41] Markiv, A., Beatson, R., Burchell, J., Durvasula, R.V. and Kang, A.S. (2011) Expression of Recombinant Multi-Coloured Fluorescent Antibodies in gor $/$ trxB $^{-}$E. coli Cytoplasm. BMC Biotechnology, 11, 117. http://dx.doi.org/10.1186/1472-6750-11-117

[42] Phillips, K.A., Marshall, D.A., Haas, J.S., Elkin, E.B., Liang, S.Y., Hassett, M.J., Ferrusi, I., Brock, J.E. and Van Bebber, S.L. (2009) Clinical Practice Patterns and Cost Effectiveness of Human Epidermal Growth Receptor 2 Testing Strategies in Breast Cancer Patients. Cancer, 115, 5166-5174. http://dx.doi.org/10.1002/cncr.24574

[43] Bevis, B.J. and Glick, B.S. (2002) Rapidly Maturing Variants of the Discosoma Red Fluorescent Protein (DsRed). Nature Biotechnology, 20, 83-87. http://dx.doi.org/10.1038/nbt0102-83

[44] Gadd, S.J. and Ashman, L.K. (1983) Binding of Mouse Monoclonal Antibodies to Human Leukaemic Cells via the Fc Receptor: A Possible Source of "False Positive” Reactions in Specificity Screening. Clinical and Experimental Immunology, 54, 811-818.

[45] Imamura, N., Ota, H. and Kuramoto, A. (1991) CD7 False-Positive Acute Myelogenous Leukemia and Promyelocytic Leukemia Cell Line HL-60: Characterization of CD7 Epitopes by Four Monoclonal Antibodies. American Journal of Hematology, 38, 72-74. http://dx.doi.org/10.1002/ajh.2830380114

[46] Magnusson, K.E., Bartonek, E., Nordkvist, E., Sundqvist, T. and Asbrink, E. (1987) Fluorescence-Linked Immunosorbent Assay (FLISA) for Quantification of Antibodies to Food Antigens. Immunological Investigations, 16, 227-240. http://dx.doi.org/10.3109/08820138709030578

[47] Velappan, N., Clements, J., Kiss, C., Valero-Aracama, R., Pavlik, P. and Bradbury, A.R. (2008) Fluorescence Linked Immunosorbant Assays Using Microtiter Plates. Journal of Immunological Methods, 336, 135-141. http://dx.doi.org/10.1016/j.jim.2008.04.007

[48] Sakamoto, S., Pongkitwitoon, B., Nakahara, H., Shibata, O., Shoyama, Y., Tanaka, H. and Morimoto, S. (2012) Fluo- 
bodies against Bioactive Natural Products and Their Application in Fluorescence-Linked Immunosorbent Assay. Antibodies, 1, 239-258. http://dx.doi.org/10.3390/antib1020239

[49] Kim, I.S., Shim, J.H., Suh, Y.T., Yau, K.Y.F., Hall, J.C., Trevors, J.T. and Lee, H. (2002) Green Fluorescent Protein-Labeled Recombinant Antibody for Detecting the Picloram Herbicide. Bioscience, Biotechnology, and Biochemistry, 66, 1148-1151. http://dx.doi.org/10.1271/bbb.66.1148

[50] Oelschlaeger, P., Srikant-Iyer, S., Lange, S., Schmitt, J. and Schmid, R.D. (2002) Fluorophor-Linked Immunosorbent Assay: A Time- and Cost-Saving Method for the Characterization of Antibody Fragments Using a Fusion Protein of a Single-Chain Antibody Fragment and Enhanced Green Fluorescent Protein. Analytical Biochemistry, 309, 27-34. http://dx.doi.org/10.1016/S0003-2697(02)00290-7

[51] Ohshima, M., Inoue, K., Hayashi, H., Tsuji, D., Mizugaki, M. and Itoh, K. (2010) Generation of AcGFP Fusion with Single-Chain Fv Selected from a Phage Display Library Constructed from Mice Hyperimmunized against 5-Methyl 2'-Deoxycytidine. Protein Engineering, Design \& Selection, 23, 881-888. http://dx.doi.org/10.1093/protein/gzq066

[52] Sakamoto, S., Taura, F., Pongkitwitoon, B., Putalun, W., Tsuchihashi, R., Kinjo, J., Tanaka, H. and Morimoto, S. (2010) Development of Sensitivity-Improved Fluorescence-Linked Immunosorbent Assay Using a Fluorescent SingleDomain Antibody against the Bioactive Naphthoquinone, Plumbagin. Analytical and Bioanalytical Chemistry, 396, 2955-2963. http://dx.doi.org/10.1007/s00216-010-3535-9

[53] Sakamoto, S., Tanizaki, Y., Pongkitwitoon, B., Tanaka, H. and Morimoto, S. (2011) A Chimera of Green Fluorescent Protein with Single Chain Variable Fragment Antibody against Ginsenosides for Fluorescence-Linked Immunosorbent Assay. Protein Expression and Purification, 77, 124-130. http://dx.doi.org/10.1016/j.pep.2011.01.010

[54] Sakamoto, S., Pongkitwitoon, B., Sasaki-Tabata, K., Putalun, W., Maenaka, K., Tanaka, H. and Morimoto, S. (2011) A Fluorescent Single Domain Antibody against Plumbagin Expressed in Silkworm Larvae for Fluorescence-Linked Immunosorbent Assay (FLISA). Analyst, 136, 2056-2063. http://dx.doi.org/10.1039/c1an15027h

[55] Ferrara, F., Listwan, P., Waldo, G.S. and Bradbury, A.R. (2011) Fluorescent Labeling of Antibody Fragments Using Split GFP. PLoS One, 6, e25727. http://dx.doi.org/10.1371/journal.pone.0025727

[56] Chudakov, D.M., Matz, M.V., Lukyanov, S. and Lukyanov, K.A. (2010) Fluorescent Proteins and Their Applications in Imaging Living Cells and Tissues. Physiological Reviews, 90, 1103-1163. http://dx.doi.org/10.1152/physrev.00038.2009

[57] Holliger, P. and Hudson, P.J. (2005) Engineered Antibody Fragments and the Rise of Single Domains. Nature Biotechnology, 23, 1126-1136. http://dx.doi.org/10.1038/nbt1142 